\title{
Microresearch: borrowing from the microfinance experience
}

$\mathrm{W}$ ho is at risk of severe head injuries in the hospital in Mbarara, and what are the outcomes? Why do some mothers in rural western Uganda seek care early for babies with severe diarrhea while others delay until the babies are in shock? These are 2 of the many vital applied health research questions raised by Ugandan health faculty members at a recent multidisciplinary research training workshop in Mbarara. Participants refined their questions, developed appropriate methodology and discussed the potential for this research to affect local health programs. But after days of hard work, a more worrisome question arose: Where will the money come from to undertake these small studies?

The lack of research capacity and access to local research funds has precluded the development of quality-of-care research using the "plan, do, study, act" approach common in industrialized countries. Local health faculty members are best placed to identify high-priority problems, help assemble local resources to study and solve problems and, most importantly, push local stakeholders into action. Motivation for change would be high, given that the community would live with the benefits or ongoing failures. Local research would also foster a culture of inquiry that would permeate the local health care system.

The scarcity of funding for locally applied research persists despite the dramatic increases in research dollars being spent in the developing world. The Bill and Melinda Gates Foundation, governments of developed countries and the World Health Organization have all invested millions of dollars in research on disease-specific health problems in developing countries, including tuberculosis, malaria and HIV/AIDS. Although this has led to important advances in health care, most projects are conceived, designed and managed by researchers from developed countries and offer little opportunity for local health faculty members to increase their research capacity. Even the Special Programme for Research and Training in Tropical Diseases (www.who.int/tdr/), which offers small grants to researchers in developing countries, does not accept applications beyond those related to infectious diseases. Furthermore, the program is run from a distance and local health faculty members are not involved in selecting the most relevant research projects.

To enhance capacity and find funding for locally applied research in developing countries, a new model is needed. Such a model might be found in the bold microfinance concept of the 2006 Nobel Peace Prize winner, Muhammad Yunus, who revolutionized financial opportunities for the poor in many developing countries. ${ }^{1}$ An analogous approach could revolutionize research opportunities in such settings. Let's call it "microresearch."

Borrowing from microfinance principles, a microresearch model would offer grants of about $\$ 3000$ to those who normally have no access to research funding opportunities. Only immediately relevant, local, applied health research questions would be eligible. Multidisciplinary groups of local faculty members would set the research priorities, assist in developing and reviewing grant applications, and assess the progress and outcomes of each project. Research networks could evolve, allowing local research groups to share new knowledge and develop best practices. Repayment of monetary loans is a measure of success in microfinance; the parallel in microresearch would be improvement in the health of communities, as assessed by community members. This approach would reward both the researchers and the communities.

Barriers, such as insufficient baseline local research knowledge and the capacity to do research, could be addressed by providing online access to clinical research education opportunities, such as those developed through the Canadian Child Health Clinician Scientist Program (www.cchcsp .ca). To ensure a high rate of success in research, prominent clinical and health services researchers worldwide could volunteer time to mentor a microresearch group.

A dedicated start-up fund of \$20-\$30 million, distributed among developing countries and managed by local universities, is needed. Bureaucracy must be kept to a minimum, and the major focus kept on the outcomes, including enhanced research capacity, answers to local research questions and regional health improvements. As with microfinance, the spinoffs for microresearch would likely be dramatic, with improved health reaching areas of the world where the need is greatest.

Making microresearch a reality will require champions to get the movement underway, perhaps as pilot projects in 2 or 3 countries. We need to persuade governments, nongovernmental organizations and volunteers to step up with the necessary financial and mentoring support. The developing world is waiting.

\section{Noni MacDonald MD MSc \\ Section Editor, Public Health \\ CMAJ}

Jerome Kabakyenga MD PhD

Dean, Faculty of Medicine

Mbarara University of Science and Technology

Mbarara, Uganda

With the Editorial-Writing Team (Paul C. Hébert MD MHSc, Rajendra Kale MD, Matthew B. Stanbrook MD PhD,

Barbara Sibbald BJ, Ken Flegel MDCM MSc and Amir Attaran LLB DPhil)

Competing interests: None declared for Jerome Kabakyenga. See www.cmaj.ca/misc/edboard.shtml for the Editorial-Writing Team's statements.

\section{REFERENCE}

1. Gangemi J. Nobel winner Yunus: microcredit missionary. Bus Week 2005 Dec 26. Available: www.businessweek.com/magazine/content/05_52/b3965024.htm (accessed 2008 Jul 24). 